\title{
REVIEW ARTICLE OPEN Nanomedicines based on nanoscale metal-organic frameworks for cancer immunotherapy
}

\author{
Xiao-fang Zhong ${ }^{1}$ and Xun Sun ${ }^{1}$
}

\begin{abstract}
Cancer immunotherapy, with an aim to enhance host immune responses, has been recognized as a promising therapeutic treatment for cancer. A diversity of immunomodulatory agents, including tumor-associated antigens, adjuvants, cytokines and immunomodulators, has been explored for their ability to induce a cascading adaptive immune response. Nanoscale metal-organic frameworks (nMOFs), a class of crystalline-shaped nanomaterials formed by the self-assembly of organic ligands and metal nodes, are attractive for cancer immunotherapy because they feature tunable pore size, high surface area and loading capacity, and intrinsic biodegradability. In this review we summarize recent progress in the development of nMOFs for cancer immunotherapy, including cancer vaccine delivery and combination of in situ vaccination with immunomodulators to reverse immune suppression. Current challenges and future perspectives for rational design of nMOF-based cancer immunotherapy are also discussed.
\end{abstract}

Keywords: cancer immunotherapy; nanoscale metal-organic frameworks (nMOFs); cancer vaccine; in situ vaccination; immunomodulators; immune response

Acta Pharmacologica Sinica (2020) 41:928-935; https://doi.org/10.1038/s41401-020-0414-6

\section{INTRODUCTION}

Over the past several decades, immunotherapy has gained significant attention as a new powerful treatment against cancer. The major goal of cancer immunotherapy is to awaken and strengthen the immune system [1-3]. Various cancer immunotherapy strategies have been investigated, such as cancer vaccines and immune checkpoint therapy [4, 5]. For example, several vaccines have been designed to target "universal" tumor antigens shared by many patients, but they have shown only modest clinical successes, in part because of weak immunogenicity [6]. Neoantigens can bind to T cell receptors (TCRs) with higher affinity than tumor-associated antigens, and they can induce more robust $T$ cell responses [7]. However, patient-specific neoantigens have yet to be identified [8].

Rather than generating the cancer vaccine in vitro from isolated neoantigens, some researchers have attempted to generate vaccines in vivo which has been termed in situ vaccination [9]. This approach avoids the need to identify and isolate neoantigens, and it can exploit the entire repertoire of antigens expressed by tumors in a given patient, allowing the development of personalized vaccines. Ideally, an in situ vaccine should induce local cancer cell death, which facilitates the release of tumor antigens, and it should enhance antigen uptake and activation of antigen-presenting cells (APCs) to elicit antitumor T cell responses [10]. These processes can be achieved through photodynamic therapies, radiotherapies and certain chemotherapies, such as anthracyclines and oxaliplatin [11].

Nanotechnology may offer unique possibilities for cancer immunotherapy because nanoparticles can serve as passive vehicles for transporting immunostimulatory agents such as antigens and adjuvants, protecting these agents from degradation and delaying their removal from the body $[12,13]$. In addition, nanomedicines that properly integrate nanotechnology can selectively target lymph nodes, immune cells, and tumor sites to improve therapeutic efficacy [14, 15].

Accordingly, various types of nanocarriers have been developed, such as liposomes [16, 17], micelles [18, 19], mesoporous silica nanoparticles [20] and nanosized metal-organic frameworks (nMOFs). nMOFs, which are hybrid porous materials built from metal ions or clusters and organic bridging ligands, have gained much popularity over the past two decades [21]. nMOFs have been designed using diverse synthetic strategies and applied to gas separation, catalysis, and energy storage and, more recently, drug delivery [22-25]. nMOFs intrinsically possess large surface areas, highly ordered porosities, and well-defined structures, which endow these materials with the capability of loading and releasing different cargos, especially therapeutic agents. The most often studied nMOFs are Materials of the Institute Lavoisier (MIL), zeolitic imidazolate frameworks (ZIFs), porous coordination networks (PCNs) and University of Oslo (UIO) nanoparticles. For example, MIL-53(Fe), composed of terephthalate anions and $\mathrm{Fe}(\mathrm{III})$ octahedra, was used to adsorb ibuprofen with a loading capacity of $20 \%$ (wt) [26]. A complete release of ibuprofen took approximately 21 days, which was proven to have zero-order kinetics. ZIF-8 has a porous polymeric network structure made up of zinc metal centers tetrahedrally coordinated to 2methylimidazole ligands, giving a sodalite topology with a surface of $\sim 1600 \mathrm{~m}^{2} / \mathrm{g}$ and a six-ring pore aperture of $3.4 \AA$.

nMOFs possess the following unique advantages that enable them to perform as promising platforms for drug delivery and

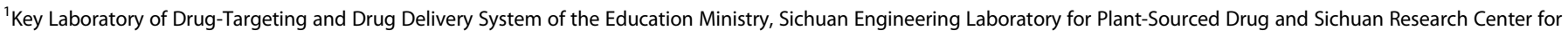
Drug Precision Industrial Technology, West China School of Pharmacy, Sichuan University, Chengdu 610064, China

Correspondence: Xun Sun (sunxun@scu.edu.cn)

Received: 10 December 2019 Revised: 29 March 2020 Accepted: 30 March 2020

Published online: 30 April 2020 
cancer immunotherapy. First, their versatile structures provide nMOFs with diverse morphologies, compositions, sizes and chemical properties, which endow them with multifunctionalities such as lymph node-targeting ability and the ability to codeliver antigens and immunomodulators. Second, large surface areas and high porosities endow nMOFs with much higher

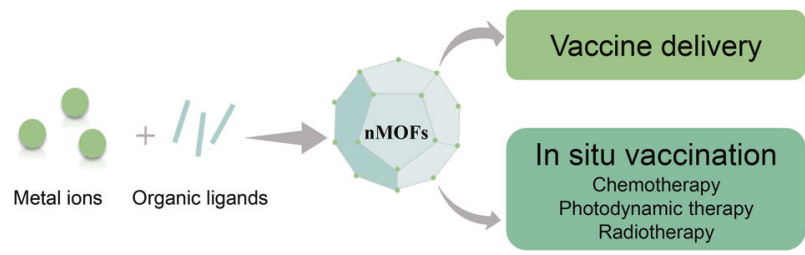

Fig. 1 Schematic illustration of the application of nanosized metal-organic frameworks (nMOFs) in cancer immunotherapy. These applications include vaccine delivery and in situ vaccination. antigen/adjuvant/cytokine loading capacity than liposomes or micelles. Third, labile metal-ligand bonds ensure nMOFs are degraded at specific sites such as endosomes/lysosomes or tumor cells, which provides relatively controlled drug release when compared with that of mesoporous silica nanoparticles [27-29]. The literature describes at least four ways in which drugs can be loaded into nMOFs: (i) physical adsorption onto the outer crystal surface, (ii) covalent conjugation to the framework, (iii) noncovalent binding within sufficiently large pores of the framework, and (iv) coprecipitation with the framework during nMOF self-assembly under physiological conditions.

These four methods have proven effective for loading nMOFs with antigens, adjuvants and immunomodulators for cancer immunotherapy [30,31]. The large surface area of nMOFs means they can accommodate large amounts of drugs or other cargo, and their tunable structure means they can easily be functionalized or modified for specific purposes, such as targeting immune

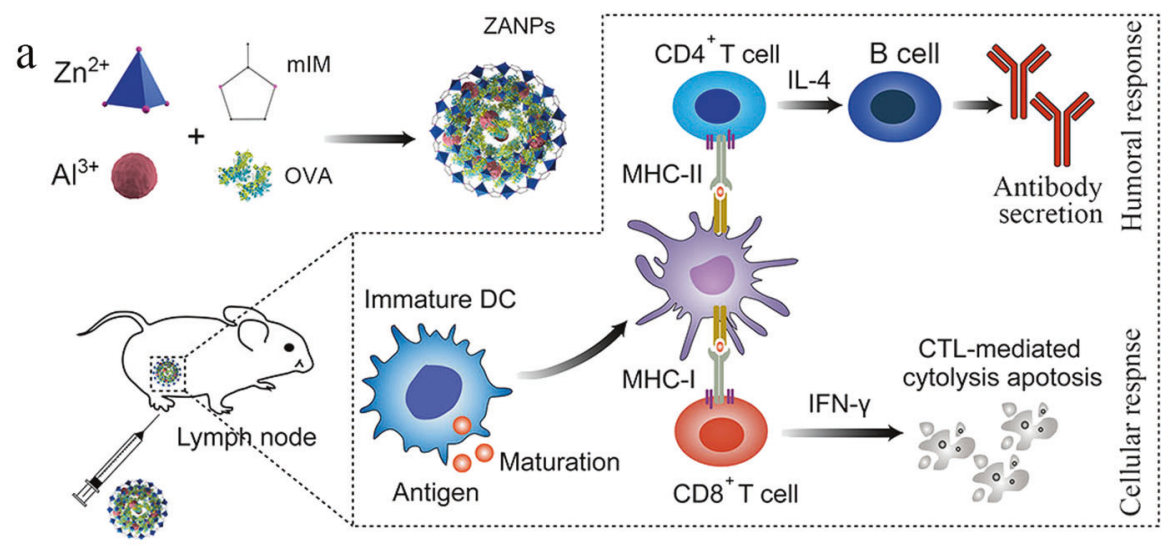

b

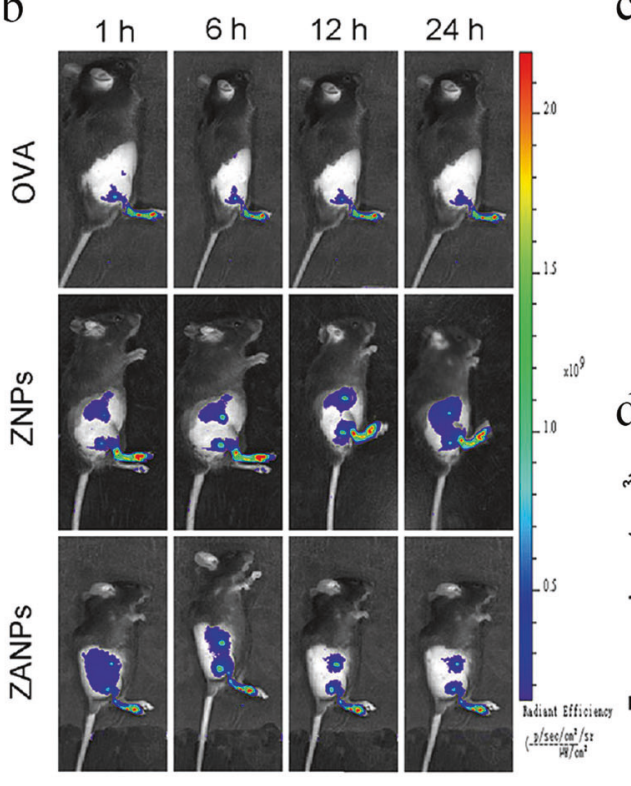

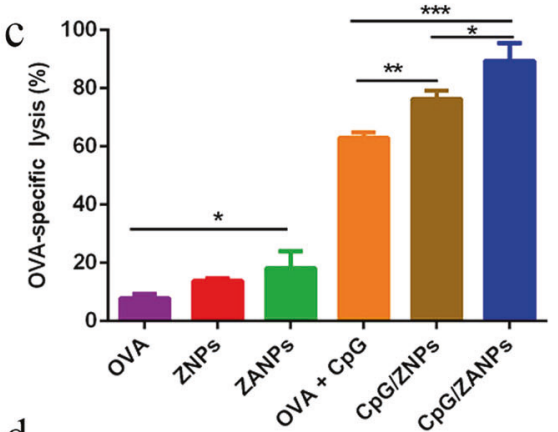

$\mathrm{d}$

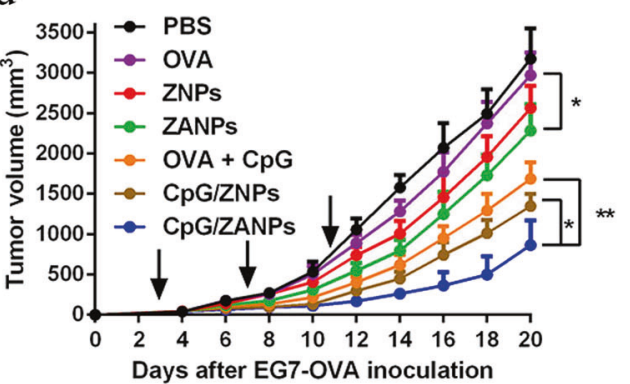

Fig. 2 Lymph node-targeting nMOFs as an efficient cancer vaccine delivery platform. a Schematic illustration of $\mathrm{Zn}^{2+}-$ based $\mathrm{nMOFs}$ containing aluminum adjuvant and OVA (ZANPs), and how they evoke efficient humoral and cellular immune responses. b In vivo nearinfrared fluorescence imaging of different formulations at 1, 6, 12 or $24 \mathrm{~h}$ after administration in the footpad. c OVA-specific CTL response elicited by different formulations, based on flow cytometry of CFSE labeling. d Tumor volume from mice challenged with EG7-OVA (EL-4 thymoma tumor cells transfected with the OVA gene) cells. ${ }^{*} P<0.05,{ }^{* *} P<0.01,{ }^{* * *} P<0.001$. CTL cytotoxic T lymphocyte, DCs dendritic cells, mIM imidazole, OVA ovalbumin, ZNP $\mathrm{Zn}^{2+}$-based nMOFs containing OVA but no adjuvant, ZANPs $\mathrm{Zn}^{2+}$-based nMOFs containing aluminum adjuvant and OVA. This figure was adapted with permission from ref. [48]. Copyright 2019 Elsevier B.V. 
930

cells. The relatively liable bonds between the metal and ligands facilitate the release of the cargo at the target site.

This review covers recent progress in using nMOFs for cancer immunotherapy. First, we illustrate the benefit of using nMOFs for the delivery of cancer vaccines. Second, we provide examples of nMOFs that serve as in situ cancer vaccines and have been simultaneously combined with immunomodulators (Fig. 1). We conclude by briefly discussing challenges and perspectives in the application of nMOFs in cancer immunotherapy.

\section{CANCER VACCINE DELIVERY BASED ON NMOFS}

nMOFs are compositionally and structurally diverse, allowing the facile synthesis of nMOFs with suitable shapes, sizes and chemical properties. Given their characteristic high loading capacity, nMOFs are exceedingly attractive as cancer vaccine vehicles to codeliver antigens and adjuvants to APCs or lymph nodes.

Codelivery of antigens and adjuvants to enhance the uptake of APCs using nMOFs

Using antigens on their own as vaccines usually elicits weak immune responses because the antigens show low immunogenicity and/or off-target effects [32]. Immune responses are usually stronger when the antigens are loaded into nanoparticles or conjugated to them. The high loading capacity of nMOFs means that they can be engineered as cancer vaccine platforms to codeliver antigens and adjuvants to enhance their detection by APCs, which process them, display them in complex with the major histocompatibility complex (MHC) molecules on their surface, and thereby activate antigen-specific $T$ cells [33]. For example, Qu and coworkers loaded ovalbumin (OVA) into ZIF-8, and cytosine-phosphate-guanine oligodeoxynucleotide (CpG ODN) was adsorbed into $200-\mathrm{nm}$ particles as an adjuvant. The nMOFs were efficiently internalized by RAW264.7 cells, and they elicited a potent memory immune response [34]. Zhang et al. loaded OVA into complexes of $\mathrm{Eu}^{2+}$ and guanine monophosphate (GMP) in one-pot coprecipitation, and CpG was allowed to adsorb via Watson-Crick base pairing. $\mathrm{Eu}^{2+}$ and GMP acted as nMOF coordinating partners. The nMOFs were taken up by RAW264.7 cells to a greater extent than OVA alone or a simple mixture of free OVA and CpG. At pH values below 5.0, the metal-ligand bonds broke, destroying the nMOFs and releasing OVA. The nMOFs triggered higher secretion of the Th1-polarizing cytokine TNF-a than OVA alone or OVA/CpG [35].

Cancer vaccines can be prophylactic (intended to prevent future infection) or therapeutic (intended to treat existing infection) [36]. Prophylactic cancer vaccines have proven effective in a few cases, such as in cancers related to human papillomavirus infection or chronic infection with hepatitis B virus [37, 38]. In contrast, developing therapeutic cancer vaccines has proven much more challenging. Therapeutic vaccines should elicit systemic immunity, especially cellular-mediated immunity. They should trigger the expansion and differentiation of antigen-specific $\mathrm{CD}^{+}{ }^{+}$cells into cytotoxic T lymphocytes (CTLs), which kill cancer cells and generate long-living $\mathrm{CD}^{+}$memory $\mathrm{T}$ cells. Vaccines can trigger these effects by delivering antigen to APCs, among which dendritic cells (DCs) are particularly effective at stimulating T cells [39]. More importantly, CD8a ${ }^{+}$DCs and/or $\mathrm{CD} 103^{+} \mathrm{DCs}$ can specifically present exogenous antigens on MHC-I molecules and thereby prime CTLs, a process called "cross-presentation" $[40,41]$. Xue and coworkers [42] developed MIL-101-Fe- $\mathrm{NH}_{2}$ to codeliver OVA and CpG in mice, which led to much higher uptake by DCs and immune responses by CTLs and other immune cells than the mixture of OVA and CpG.

Vaccine vehicles for lymph node targeting

Lymph nodes contain many phagocytically active DCs, including lymph node-resident $C D 8^{+} \mathrm{DCs}$, and they are therefore the main sites of immune activation and surveillance [41, 43, 44]. Studies have confirmed that delivering antigens to lymph nodes can improve antigen-specific adaptive immune responses [45, 46]. The size of nanovesicles appears to be the most important factor for lymph node targeting: particles smaller than $100 \mathrm{~nm}$ are more likely to drain into lymph nodes [47]. nMOFs are compositionally and structurally diverse, allowing the facile synthesis of nMOFs with suitable shapes, sizes and chemical properties for lymph node targeting.

Taking advantage of biomineralization, Sun et al. loaded aluminum adjuvant integrated-ZIF-8 with OVA, and they coated the nanoparticles with CpG via electrostatic interaction to boost Th1-type immunity. The resulting 80-nm nanoparticles (CpG/ ZANPs) efficiently codelivered OVA and CpG to lymph nodes, where the same DCs phagocytosed both antigen and adjuvant (Fig. 2a, b). Within the acidic lysosomes, the imidazole in ZIF-8 became protonated, disrupting its interaction with zinc ions, thereby allowing OVA to escape from lysosomes into the cytoplasm and be cross-presented by DCs. These nanoparticles induced a greater proportion of $\mathrm{CD}^{+}$T cells secreting IFN- $\gamma^{+}$as well as higher lgG2a antibody secretion than the mixture of OVA and $\mathrm{CpG}$. Consistent with the enhanced antigen-specific CTL response, CpG/ZANPs significantly inhibited tumor growth and prolonged survival in mice bearing EG7-OVA cells (EL-4 thymoma tumor cells transfected with the OVA gene) (Fig. 2c, d).

Taken together, these results show that the nMOF-based vaccine delivery system offers the following advantages: (i) nMOFs have an extremely high antigen loading capacity; (ii) nMOFs can increase antigen immunogenicity and uptake by APCs; (iii) the nMOFs degraded in the acidic endo/lysosomes enable release of the encapsulated antigens into the cytoplasm, leading to enhanced antigen cross-presentation ability; and (iv) the compatibility of nMOFs with various metal ions and organic linkers allows tailoring of the system to different target tissues, such as lymph nodes.

\section{IN SITU CANCER VACCINATION WITH NMOFS FOR COMBINATORIAL THERAPY}

To be effective, cancer vaccines should elicit immune responses to antigens expressed by tumors. To achieve this goal, the past several decades in cancer vaccination have been characterized by considerable effort into the discovery of tumor-associated antigens. However, these tumor-associated antigen-based vaccines have proven ineffective in animal models and clinical trials. An alternative is to design vaccines based on neoantigens, which are solely expressed in cancer and not normal cells. Therefore, neoantigen-based cancer vaccines may be more specific, more effective and less toxic than vaccines targeting tumor-associated antigens. However, neoantigens are quite difficult to isolate, and the best approach for predicting them is unknown [49, 50].

Instead, researchers have begun to explore vaccines formed in situ based on the full array of the patient's tumor antigens. The concept of in situ vaccination involves any approach that exploits antigens available at a tumor site to induce a tumor antigenspecific adaptive immune response. Tumor cells are killed by chemotherapy, radiotherapy or photodynamic therapy to release antigens, which recruit APCs to the tumor, and these APCs take up and process the antigens while being trafficked to tumor-draining lymph nodes. There, the APCs activate CTLs, which invade tumors and destroy antigen-expressing tumor cells [49, 51]. Such an approach uses antigens of patients themselves, thereby eliminating the need to previously identify and isolate the neoantigens [52-54]. Moreover, the antitumor response can be strengthened by simultaneously applying other immunotherapies $[55,56]$. Recently, nMOFs have been investigated as a possible vehicle for effective in situ cancer vaccination, including in concert with chemotherapy, photodynamic therapy and radiotherapy. 


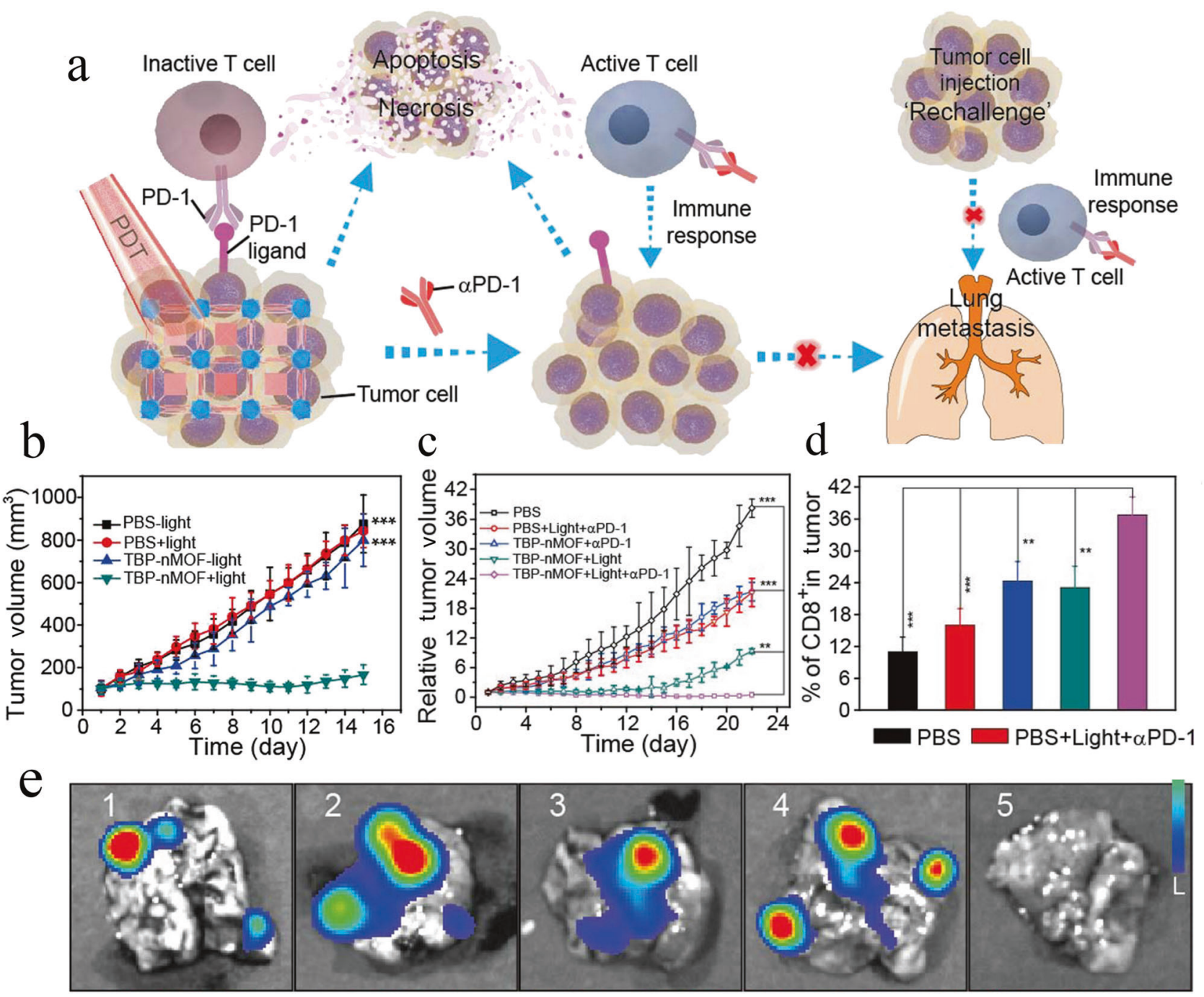

Fig. $3 \mathrm{~A} \mathrm{Zr}_{6}$-connected nMOF integrating a benzoporphyrin-based photosensitizer (TBP-nMOF) for photodynamic treatment (PDT) and combination immunotherapy. a Proposed mechanism of antitumor immune responses induced by TBP-nMOF, and the synergy between photodynamic therapy and anti-PD-1 antibody to inhibit tumor metastasis. b, c Tumor volume changes after b photodynamic therapy (light) alone or c combined PDT with antibody against PD-1 ( $\alpha$-PD-1). $\mathbf{d}$ Percentage of $\mathrm{CD}^{+} \mathrm{T}$ cells that infiltrated the tumors after the treatments in c. e Bioluminescence images of the lung from mice bearing luciferase-expressing primary 4T1 tumors on the right back of hind leg region after various treatment; 1: PBS; 2: PBS + light $+\alpha$-PD-1; 3: TBP-nMOF $+\alpha-$ PD-1; 4: TBP-nMOF + light; 5: TBP-nMOF + light $+\alpha-P D-1 .{ }^{* * P}<0.01$, ${ }^{* * *} P<0.001$. This figure was adapted with permission from ref. [70]. Copyright 2018 American Chemical Society.

Chemical drug-mediated in situ vaccination using nMOFs It was reported that chemotherapy could be used for in situ vaccination to promote antitumor immune responses. Certain chemotherapeutics, such as the anthracyclines doxorubicin, idarubicin and oxaliplatin, can induce so-called immunogenic cell death, which releases calreticulin, ATP and high-mobility group box protein 1 (HMGB1) into the extracellular milieu [57]. These damage-associated molecular patterns (DAMPs) induce death and apoptosis of tumor cells as well as their engulfment by DCs, increasing the number of $\mathrm{T}$ lymphocytes and the ratio of $\mathrm{CD}^{+}$ CTLs to FOXP3 ${ }^{+}$regulatory T cells [58-60].

Lin and coworkers encapsulated doxorubicin and catalase into ZIF-8 nanoparticles, which were then coated with cell membrane from murine melanoma cells. This tumor cell "coating" allowed the nMOFs to escape immune surveillance and accumulate in tumor tissues. Since doxorubicin triggers immunogenic cell death, these coated nMOFs increased the number of antigen-specific $\mathrm{CD}^{+}{ }^{+} \mathrm{CD} 8^{+}$tumor-infiltrating $T$ cells. At the same time, the nMOFs downregulated the expression of hypoxia-inducible factor-1a, reducing the risk of drug resistance and immune escape [61]. As PD-L1 expressed by cancer cells can actually inhibit antigenspecific CTLs from recognizing and killing tumor cells and even drive CTLs into apoptosis, these coated nMOFs were combined with in situ vaccination with a monoclonal antibody against PD-1. This approach increased the number of infiltrating $\mathrm{CD}^{+} \mathrm{T}$ cells and the production of interleukin-12 and tumor necrosis factor- $a$, inhibiting the growth and metastasis of murine melanoma tumor cells in mice [62].

Photodynamic therapy-mediated in situ vaccination using nMOFs Photodynamic therapy involves exposing a photosensitizer to visible or near-infrared light to generate reactive oxygen species (ROS), particularly singlet oxygen, which cause necrosis or apoptosis of tumor cells. The resulting release of DAMPs activates macrophages and DCs, which migrate to lymph nodes, where they activate humoral and cell-mediated immune responses [63, 64]. In parallel, photodynamic therapy triggers the release of inflammatory mediators and cytokines into the tumor, stimulating an 

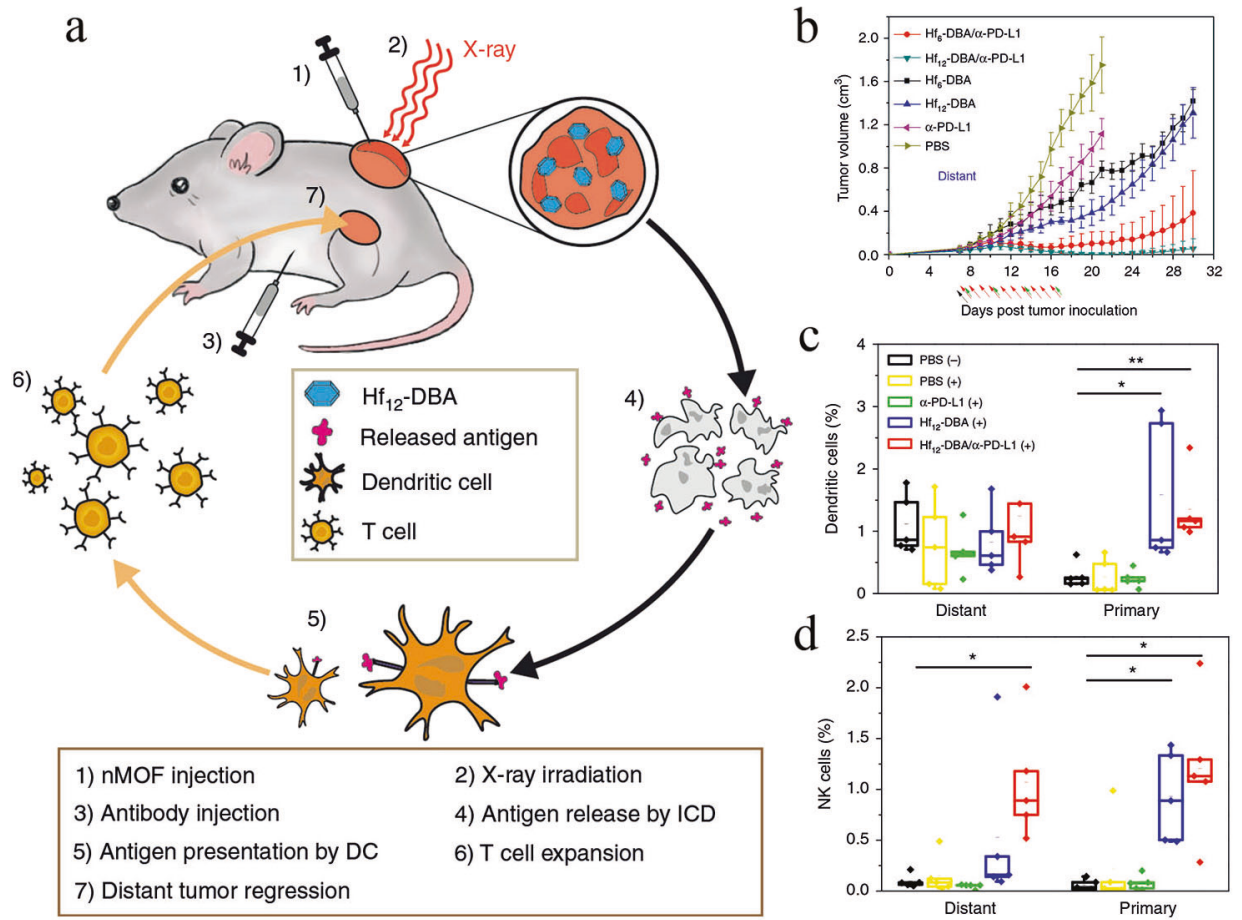

Fig. 4 An Hf-based nMOF serve as radioenhancer, integrated low-dose radiation for immune response stimulation. a Abscopal effect of nMOF-enhanced radiotherapy: Hf-based nMOF triggers immunogenic cell death, which releases tumor antigen serve as in situ vaccine, while anti-PD-1 antibody reverses the immunosuppressive tumor microenvironment, enhancing $\mathrm{T}$ cell expansion and tumor infiltration. DC, dendritic cells. b Growth of distant tumors after mice were injected different formulations bilaterally on the right back of hind leg region with CT26 colorectal tumor cells. c, d Primary and distant tumors were collected and analyzed for content of tumor-infiltrating DCs natural killer (NK) cells. This figure was adapted with permission from ref. [80]. Copyright 2018 Springer Nature.

adaptive immune response [65]. A powerful demonstration of this type of in situ vaccination is found in mice: mice vaccinated with lysates of photodynamically treated (Photofrin as photosensitizer) tumor cells resisted challenge with the same tumor type. Subsequent studies have revealed that other photosensitizers can also be used for generating in situ vaccines $[66,67]$. This effective response seems to require activation of $\mathrm{CD}^{+} \mathrm{T}$ cells $[68]$.

Although various photosensitizers can be effective for in situ vaccination mediated by photodynamic therapy, most of them show low aqueous solubility or poor photostability. To address these limitations, MIL-100 (Fe) was used to encapsulate different kinds of photosensitizers, notably 2-((4'-(2,2-bis(4-methoxyphenyl)-1-phenylvinyl)-[1,1'-biphenyl]- 4-yl)(phenyl)methylene) malononitrile (TPEDC), (E)-2-(4-(4-(2,2-bis(4-methoxyphenyl)-1phenylvinyl)styryl)-3-cyano-5,5-dimethylfuran-2(5H)-ylidene)malononitrile (TPETCF) and chlorin e6 (Ce6) [69]. As they contain iron (III), MIL-100 ( $\mathrm{Fe}$ ) nanoparticles could catalyze the decomposition of $\mathrm{H}_{2} \mathrm{O}_{2}$ into $\mathrm{O}_{2}$, which relieves tumor hypoxia and potentiates photodynamic therapy. Zhang et al. [70] integrated a tetra( $p$ benzoato)porphyrin-based photosensitizer into a $\mathrm{Zr}_{6}$-connected nMOF measuring $100 \mathrm{~nm}$ (Fig. 3a). The TBP-nMOF showed stronger infrared luminescence and redshifted absorption than traditional porphyrin-based MOFs, and it generated much higher amounts of singlet oxygen even under low oxygen concentrations. The benzoporphyrin-containing nMOF not only induced the apoptosis of 4T1 murine breast cancer cells but also stimulated a strong increase in the number of tumor-infiltrating $\mathrm{CD}^{+}$and $\mathrm{CD}^{+} \mathrm{T}$ cells, and combining it with an antibody against PD-1 led to complete tumor elimination without recurrence in 4T1-bearing mice (Fig. 3b-d). The combination of photodynamic therapy and an antibody against PD-1 synergistically recruited tumor- infiltrating lymphocytes and inhibited metastasis of 4T1 tumors to the lungs (Fig. 3e).

Lin and his colleagues integrated benzoporphyrin with $\mathrm{Fe}_{3} \mathrm{O}$ as the metal clusters to construct $\mathrm{Fe}_{3} \mathrm{O}$-based nMOFs measuring 100 $\mathrm{nm}$. This nMOF was taken up efficiently by CT26 cells, and it decreased $\mathrm{H}_{2} \mathrm{O}_{2}$ levels through a Fenton-like reaction and downregulated hypoxia-inducible factor- $1 \mathrm{a}$, showing that it could alleviate hypoxia in tumor tissues. After photodynamic treatment, this $\mathrm{Fe}_{3} \mathrm{O}$-based nMOF caused tumor cell death effectively through immunogenic cell death, which was investigated by detecting cell surface exposure of calreticulin. Combining the nMOF with photodynamic therapy led to tumor regression, and the further addition of anti-PD-L1 antibody significantly expanded $\mathrm{CD}^{+}$and $\mathrm{CD}^{+}$CTL populations. This reversed PD-L1-mediated immunosuppression, causing complete regression of primary tumors and $90 \%$ regression of metastatic tumors in mice [71].

Through solvothermal reactions between benzoporphyrin and $\mathrm{WCl}_{6}$, benzoporphyrin was incorporated into a $\mathrm{W}$-based nMOF [72]. Anionic CpG as an adjuvant was adsorbed on the surface of the cationic nMOF. Photodynamic therapy triggered the release of antigens from tumors, while $\mathrm{CpG}$ promoted antigen internalization and presentation by DCs, which were reflected in the upregulation of MHC-II and costimulatory CD86 molecules. The resulting immune response was strong, as reflected by increases in IFN- $a$ and IL-6. Further addition of an antibody against PD-L1 led to tumor regression in a mouse model of bilateral breast cancer.

Radiotherapy-mediated in situ vaccination using nMOFs Radiotherapy destroys tumor tissue using ionizing radiation, which generates damaging hydroxyl radicals in an X-ray dose-dependent manner [73]. Immunogenic cell death induces tumor-associated 
antigen release and translocation of calreticulin to the tumor cell membrane, which acts as an "eat me" signal to DCs, ultimately activating intratumoral DCs that upregulate the costimulatory molecules CD86 and CD70. This makes tumor antigens available for cross-presentation on MHC-I molecules, where they can prime tumor-specific T cells [74, 75].

Radiotherapy promotes the delivery of not only tumorassociated antigens but also tumor DNA to DCs, which activates type I IFN production via the "stimulator of interferon genes" (STING) pathway $[76,77]$. At the same time, radiotherapy induces the production of chemokines, which recruit effector $\mathrm{T}$ cells to tumors. Emerging evidence indicates that radiotherapy can convert tumors into an in situ vaccine, and combining radiotherapy with immunotherapy will stimulate a systemic immune response to reject tumor cells [78].

As lower doses of radiation often fail to elicit sufficiently strong immune responses but higher doses can injure off-target tissues, researchers have explored nMOFs as radioenhancers to combine with low-dose radiation. The released antigens serve as in situ individualized tumor vaccines to synergize with immune checkpoint inhibitors to inhibit tumor growth [79]. For example, nMOFs were studied as an in situ vaccine in combination with immune checkpoint inhibitors with a low radiation dose to achieve systemic rejection of colorectal tumors in mouse models [80]. A $\mathrm{Hf}$-based nMOF was synthesized through coordination between $\mathrm{Hf}_{12} \mathrm{O}_{8}(\mathrm{OH})_{14}$ and 2,5-di(p-benzoato)aniline (Fig. 4a). This nMOF led to much higher production of hydroxyl radicals than $\mathrm{HfO}_{2}$, indicating that it may serve as a radioenhancer since hydroxyl radicals are the major cytotoxic radical species induced by ionizing radiation. The $\mathrm{Hf}$ nMOF-mediated radiation treatment caused immunogenic cell death, reflected in the expression of calreticulin and HMGB1, and substantially improved the ability of Hf to shrink CT26 colorectal xenografts in mice (Fig. 4b). Combining the Hf nMOF-mediated radiotherapy with an anti-PD-L1 antibody not only inhibited local irradiated tumors but also shrank distant, nontreated tumors (Fig. 4b). Flow cytometry analysis of excised tumors showed increased numbers of IFN- $\gamma$-producing $\mathrm{CD}^{+}$and $\mathrm{CD}^{+}{ }^{\mathrm{T}}$ cells. Primary tumors contained higher percentages of DCs and natural killer cells than did metastases (Fig. 4c, d), suggesting that both cell types were recruited to tumors after radiation.

Similarly, Lin and his coworkers reported an nMOF constructed from a chlorin derivative, 5,10,15,20-tetra( $p$-benzoato)chlorin, which could absorb X-ray photons and induce the emission of photoelectrons via the photoelectric effect. When the pores of this nMOF were loaded with an inhibitor of indoleamine 2,3dioxygenase and the nMOF was administered together with low-dose radiotherapy, the growth of primary and metastatic CT26 tumors was slowed in mice. Dioxygenase is overexpressed in tumor cells, where it degrades tryptophan and produces kynurenine, leading to $\mathrm{T}$ cell anergy and apoptosis, helping tumors evade the immune system [81]. This synergistic treatment increased the numbers of tumor-infiltrating $\mathrm{CD} 45^{+} \mathrm{DCs}$ and macrophages [82].

These various examples illustrate how combining appropriate nMOFs with radiotherapy can make them efficient in situ vaccines, which can synergize with immunomodulators to amplify systemic antitumor immunity.

\section{SUMMARY AND PERSPECTIVES}

Immunotherapies based on nMOFs have shown strong promise for cancer treatment. These therapies fundamentally activate an immune response through two mechanisms. One is to directly deliver tumor-associated antigens and Toll-like receptor agonists to APCs such as DCs and macrophages. This promotes APC maturation, antigen cross-presentation and $T$ cell priming. The other mechanism is to deliver drugs or apply light or X-ray radiation to allow in situ cancer vaccination. Both mechanisms promote antigen presentation and stimulate $\mathrm{T}$ cell proliferation and differentiation into CTLs as well as secretion of IFN- $\gamma$, TNF- $a$ and other cytokines.

Although nMOF-based immunotherapy has progressed substantially in the past several years, many chemical and immunological challenges stand in the way of clinical application. Undoubtedly, the most important challenges are the risk of undesired cytotoxic and genotoxic effects. Indeed, cytotoxicity was observed to strongly depend on the nMOF composition, such as the nature of the metal and organic building blocks. The oral lethal dose $50\left(L_{50}\right)$ of Fe is $30 \mathrm{~g} / \mathrm{kg}$, while that of $\mathrm{Zn}$ is $350 \mu \mathrm{g} / \mathrm{kg}$, and that of $Z r$ is $4.1 \mathrm{~g} / \mathrm{kg}[83,84]$. For organic building blocks, the $\mathrm{LD}_{50}$ values of terephthalic acid, trimesic acid, 1-methylimidazole and 2-methylimidazole are 5, 8.4, 1.13 and $1.4 \mathrm{~g} / \mathrm{kg}$, respectively. In addition, the hydrophobic-hydrophilic balance is also an important parameter [83]. When designing nMOFs, the dosage of metal ions and organic ligands needs to be considered. In addition to these organic compounds, endogenous biomolecules such as nucleotides and phospholipids can be used as ligands to reduce the risk of toxicity $[35,85]$. Most safety studies of nMOFs have examined cell lines in vitro. Thus, studies of nMOF absorption, distribution, metabolism and elimination in vivo are urgently needed.

Another challenge to the clinical application of nMOFs is inadequate stability or rapid degradation. Many studies on the stabilities of nMOFs have been conducted in water, but stability under simulated physiological conditions has been less pursued. For example, MIL-101 (Fe) possesses poor stability in phosphate buffer, while MIL-100 (Fe) is stable in water but decomposes after several days [86]. Recently, Cheng et al. [30] coated extracellular vesicles on the surface of nanosized ZIF-8 and found that biomineralized nMOFs retained their nanostructure in PBS $(\mathrm{pH}=7.4)$ but degraded in acidic buffer $(\mathrm{pH}=5.0)$. Decomposition of nMOFs at a desired region is needed for responsive drug release. Differences in degradability can be achieved by selecting different metal ions, organic linkers, and crystalline structures. Thus, the degradation time could be adjusted from a few hours to several weeks [87, 88]. However, the degradation mechanisms of nMOFs in vitro and in vivo require further study.

\section{ACKNOWLEDGEMENTS}

We acknowledge the financial support of the National Natural Science Foundation of China (Nos. 81872814 and 81673362), Sichuan Veterinary Medicine and Drug Innovation Group of China Agricultural Research System (CARS-SVDIP), the Science \& Technology Major Project of Sichuan Province (2018SZDZX0018) and the Fundamental Research Funds for the Central Universities.

\section{ADDITIONAL INFORMATION}

Competing interests: The authors declare no competing interests.

\section{REFERENCES}

1. Mellman I, Coukos G, Dranoff G. Cancer immunotherapy comes of age. Nature. 2011;480:480-9.

2. Ribas A, Wolchok JD. Cancer immunotherapy using checkpoint blockade. Science. 2018;359:1350-5.

3. Rosenberg SA, Yang JC, Restifo NP. Cancer immunotherapy: moving beyond current vaccines. Nat Med. 2004;10:909-15.

4. Wang $\mathrm{H}$, Mooney DJ. Biomaterial-assisted targeted modulation of immune cells in cancer treatment. Nat Mater. 2018;17:761-72.

5. Wei SC, Duffy CR, Allison JP. Fundamental mechanisms of immune checkpoint blockade therapy. Cancer Discov. 2018;8:1069-86.

6. Xu Z, Ramishetti S, Tseng YC, Guo S, Wang Y, Huang L. Multifunctional nanoparticles co-delivering Trp2 peptide and CpG adjuvant induce potent cytotoxic Tlymphocyte response against melanoma and its lung metastasis. J Control Release. 2013;172:259-65.

7. Hagan T, Vincent B, Wang A. Emerging nano-/microapproaches for cancer Immunotherapy. Adv Sci. 2019;6:1801847. 
8. Bethune MT, Joglekar AV. Personalized T cell-mediated cancer immunotherapy: progress and challenges. Curr Opin Biotechnol. 2017;48:142-52.

9. Lizotte PH, Wen AM, Sheen MR, Fields J, Rojanasopondist P, Steinmetz NF, et al. In situ vaccination with cowpea mosaic virus nanoparticles suppresses metastatic cancer. Nat Nanotechnol. 2016;11:295-303.

10. Hammerich L, Bhardwaj $\mathrm{N}$, Kohrt $\mathrm{HE}$, Brody JDJI. In situ vaccination for the treatment of cancer. Immunotherapy. 2016;8:315-30.

11. Zitvogel L, Kepp O, Senovilla L, Menger L, Chaput N, Kroemer G. Immunogenic tumor cell death for optimal anticancer therapy: the calreticulin exposure pathway. Clin Cancer Res. 2010;16:3100-4.

12. Smith DM, Simon JK, Baker JR Jr. Applications of nanotechnology for immunology. Nat Rev Immunol. 2013;13:592-605.

13. Velu V, Titanji K, Zhu BG, Husain S, Pladevega A, Lai LL, et al. Enhancing SIVspecific immunity in vivo by PD-1 blockade. Nature. 2009;458:206-U5.

14. Jiang $H$, Wang $Q$, Li L, Zeng $Q$, Li H, Gong $T$, et al. Turning the old adjuvant from gel to nanoparticles to amplify CD8+ T cell responses. Adv Sci. 2018;5:1700426.

15. Mi Y, Hagan IvCT, Vincent BG, Wang AZ. Emerging nano-/microapproaches for cancer immunotherapy. Adv Sci. 2019;6:1801847.

16. van Broekhoven $\mathrm{CL}$, Parish $\mathrm{CR}$, Demangel C, Britton WJ, Altin JG. Targeting dendritic cells with antigen-containing liposomes: a highly effective procedure for induction of antitumor immunity and for tumor immunotherapy. Cancer Res. 2004;64:4357.

17. Qiao C, Liu J, Yang J, Li Y, Weng J, Shao Y, et al. Enhanced non-inflammasome mediated immune responses by mannosylated zwitterionic-based cationic liposomes for HIV DNA vaccines. Biomaterials. 2016;85:1-17.

18. Zeng $\mathrm{Q}$, $\mathrm{Li} \mathrm{H}$, Jiang $\mathrm{H}, \mathrm{Yu} J$, Wang $\mathrm{Y}, \mathrm{Ke} \mathrm{H}$, et al. Tailoring polymeric hybrid micelles with lymph node targeting ability to improve the potency of cancer vaccines. Biomaterials. 2017;122:105-13.

19. Li H, Li Y, Wang X, Hou Y, Hong X, Gong T, et al. Rational design of polymeric hybrid micelles to overcome lymphatic and intracellular delivery barriers in cancer immunotherapy. Theranostics. 2017;7:4383-98.

20. Li WA, Lu BY, Gu L, Choi Y, Kim J, Mooney DJ. The effect of surface modification of mesoporous silica micro-rod scaffold on immune cell activation and infiltration. Biomaterials. 2016;83:249-56.

21. Wu MX, Yang YW. Metal-organic framework (MOF)-based drug/cargo delivery and cancer therapy. Adv Mater. 2017;29:1606134.

22. Liang J, Liang ZB, Zou RQ, Zhao YL. Heterogeneous catalysis in zeolites, mesoporous silica, and metal-organic frameworks. Adv Mater. 2017;29,1701139

23. Rui K, Wang X, Du M, Zhang Y, Wang Q, Ma Z, et al. Dual-function metal-organic framework-based wearable fibers for gas probing and energy storage. ACS Appl Mater Interfaces. 2018;10:2837-42.

24. Della Rocca J, Liu D, Lin W. Nanoscale metal-organic frameworks for biomedical imaging and drug delivery. Acc Chem Res. 2011;44:957-68.

25. Cai W, Chu CC, Liu G, Wáng YXJ. Metal-organic framework-based nanomedicine platforms for drug delivery and molecular imaging. Small. 2015;11:4806-22.

26. Horcajada P, Serre C, Maurin G, Ramsahye NA, Balas F, Vallet-Regí M, et al. Flexible porous metal-organic frameworks for a controlled drug delivery. J Am Chem Soc. 2008;130:6774-80

27. Horcajada P, Chalati T, Serre C, Gillet B, Sebrie C, Baati T, et al. Porous metalorganic-framework nanoscale carriers as a potential platform for drug delivery and imaging. Nat Mater. 2010;9:172-8.

28. Kong XQ, Deng HX, Yan FY, Kim J, Swisher JA, Smit B, et al. Mapping of functional groups in metal-organic frameworks. Science. 2013;341:882-5.

29. Li H, Eddaoudi M, O'Keeffe M, Yaghi OM. Design and synthesis of an exceptionally stable and highly porous metal-organic framework. Nature. 1999;402:276-9.

30. Cheng G, Li W, Ha L, Han X, Hao S, Wan Y, et al. Self-Assembly of extracellular vesicle-like metal-organic framework nanoparticles for protection and intracellular delivery of biofunctional proteins. J Am Chem Soc. 2018;140:7282-91.

31. Wang Z, Fu Y, Kang Z, Liu X, Chen N, Wang Q, et al. Organelle-specific triggered release of immunostimulatory oligonucleotides from intrinsically coordinated DNA-metal-organic frameworks with soluble exoskeleton. J Am Chem Soc. 2017;139:15784-91.

32. Fan YC, Moon JJ. Nanoparticle drug delivery systems designed to improve cancer vaccines and immunotherapy. Vaccines. 2015;3:662-85.

33. Miao YB, Pan WY, Chen KH, Wei HJ, Mi FL, Lu MY, et al. Engineering a nanoscale Al-MOF-armored antigen carried by a "Trojan Horse"-like platform for oral vaccination to induce potent and long-lasting immunity. Adv Funct Mater. 2019;29:1904828.

34. Zhang Y, Wang FM, Ju EG, Liu Z, Chen ZW, Ren JS, et al. Metal-organicframework-based vaccine platforms for enhanced systemic immune and memory response. Adv Funct Mater. 2016;26:6454-61.

35. Duan F, Feng XC, Yang XJ, Sun WT, Jin Y, Liu HF, et al. A simple and powerful codelivery system based on $\mathrm{pH}$-responsive metal-organic frameworks for enhanced cancer immunotherapy. Biomaterials. 2017;122:23-33.
36. Palucka K, Banchereau J, Mellman I. Designing vaccines based on biology of human dendritic cell subsets. Immunity. 2010;33:464-78.

37. Lowy DR, Schiller JT. Prophylactic human papillomavirus vaccines. J Clin Invest. 2006:116:1167-73.

38. Kao JH, Chen DS. Global control of hepatitis B virus infection. Lancet Infect Dis. 2002;2:395-403.

39. Banchereau J, Steinman RM. Dendritic cells and the control of immunity. Nature. 1998;392:245-52.

40. Eisenbarth SC. Dendritic cell subsets in T cell programming: location dictates function. Nat Rev Immunol. 2019;19:89-103.

41. Shortman K, Heath WR. The $\mathrm{CD}^{+}$dendritic cell subset. Immunol Rev. 2010;234:18-31.

42. Yang Y, Chen Q, Wu JP, Kirk TB, Xu J, Liu Z, et al. Reduction-responsive codelivery system based on a metal-organic framework for eliciting potent cellular immune response. ACS Appl Mater Interfaces. 2018;10:12463-73.

43. Joffre OP, Segura E, Savina A, Amigorena S. Cross-presentation by dendritic cells. Nat Rev Immunol. 2012;12:557-69.

44. Segura E, Amigorena S. Cross-presentation in mouse and human dendritic cells. Adv Immunol. 2015;127:1-31.

45. Liu H, Moynihan KD, Zheng Y, Szeto GL, Li AV, Huang B, et al. Structure-based programming of lymph-node targeting in molecular vaccines. Nature. 2014:507:519-22.

46. Reddy ST, van der Vlies AJ, Simeoni E, Angeli V, Randolph GJ, O'Neil CP, et al. Exploiting lymphatic transport and complement activation in nanoparticle vaccines. Nat Biotechnol. 2007;25:1159-64.

47. Jiang $H$, Wang $Q$, Sun $X$. Lymph node targeting strategies to improve vaccination efficacy. J Control Release. 2017;267:47-56.

48. Zhong XF, Zhang $Y T$, Tan $L$, Zheng $T$, Hou $Y Y$, Hong $X Y$, et al. An aluminum adjuvant-integrated nano-MOF as antigen delivery system to induce strong humoral and cellular immune responses. J Control Release. 2019;300:81-92.

49. Brody JD, Ai WZ, Czerwinski DK, Torchia JA, Levy M, Advani RH, et al. In situ vaccination with a TLR9 agonist induces systemic lymphoma regression: a phase I/II study. J Clin Oncol. 2010;28:4324-32.

50. Srivastava PK. Immunotherapy of human cancer: lessons from mice. Nat Immunol. 2000;1:363-6.

51. Hammerich L, Binder A, Brody JD. In situ vaccination: cancer immunotherapy both personalized and off-the-shelf. Mol Oncol. 2015;9:1966-81.

52. Lawler SE, Speranza MC, Cho CF, Chiocca EA. Oncolytic viruses in cancer treatment: a review. JAMA Oncol. 2017;3:841-9.

53. Sheen MR, Fiering S. In situ vaccination: Harvesting low hanging fruit on the cancer immunotherapy tree. Wiley Interdiscip Rev Nanomed Nanobiotechnol. 2019;11:e1524-e.

54. Hammerich L, Bhardwaj N, Kohrt HE, Brody JD. In situ vaccination for the treatment of cancer. Immunotherapy. 2016;8:315-30.

55. Wang C, Wang J, Zhang X, Yu S, Wen D, Hu Q, et al. In situ formed reactive oxygen species-responsive scaffold with gemcitabine and checkpoint inhibitor for combination therapy. Sci Transl Med. 2018;10:eaan3682.

56. Locy p, de Mey S, de Mey W, De Ridder M, Thielemans K, Maenhout SK. Immunomodulation of the tumor microenvironment: turn foe into friend. Front Immunol. 2018;9:2909.

57. Hodge JW, Garnett CT, Farsaci B, Palena C, Tsang KY, Ferrone $\mathrm{S}$, et al. Chemotherapy-induced immunogenic modulation of tumor cells enhances killing by cytotoxic T lymphocytes and is distinct from immunogenic cell death. Int J Cancer. 2013;133:624-36

58. Kroemer G, Galluzzi L, Kepp O, Zitvogel L. Immunogenic cell death in cancer therapy. Annu Rev Immunol. 2013;31:51-72.

59. Krysko DV, Garg AD, Kaczmarek A, Krysko O, Agostinis $P$, Vandenabeele $P$. Immunogenic cell death and DAMPs in cancer therapy. Nat Rev Cancer. 2012;12:860-75.

60. Cook AM, Lesterhuis WJ, Nowak AK, Lake RA. Chemotherapy and immunotherapy: mapping the road ahead. Curr Opin Immunol. 2016;39:23-9.

61. Noman MZ, Desantis G, Janji B, Hasmim M, Karray S, Dessen P, et al. PD-L1 is a novel direct target of HIF-1a, and its blockade under hypoxia enhanced MDSCmediated T cell activation. J Exp Med. 2014;211:781.

62. Zou MZ, Liu WL, Li CX, Zheng DW, Zeng JY, Gao F, et al. A multifunctional biomimetic nanoplatform for relieving hypoxia to enhance chemotherapy and inhibit the PD-1/PD-L1 axis. Small. 2018; 14:e1801120.

63. Mroz $P$, Hashmi JT, Huang YY, Lange N, Hamblin MR. Stimulation of anti-tumor immunity by photodynamic therapy. Expert Rev Clin Immunol. 2011;7:75-91.

64. Agostinis P, Berg K, Cengel KA, Foster TH, Girotti AW, Gollnick SO, et al. Photodynamic therapy of cancer: an update. CA Cancer J Clin. 2011;61:250-81.

65. Allison RR, Moghissi K. Photodynamic therapy (PDT): PDT mechanisms. Clin Endosc. 2013:46:24-9.

66. Korbelik M, Stott B, Sun J. Photodynamic therapy-generated vaccines: relevance of tumour cell death expression. Br J Cancer. 2007;97:1381-7. 
67. Korbelik M, Sun J. Photodynamic therapy-generated vaccine for cancer therapy. Cancer Immunol Immunother. 2006;55:900-9.

68. Kabingu E, Vaughan L, Owczarczak B, Ramsey KD, Gollnick SO. CD8 ${ }^{+}$T cellmediated control of distant tumours following local photodynamic therapy is independent of CD4+ T cells and dependent on natural killer cells. Br J Cancer. 2007:96:1839-48.

69. Hu F, Mao D, Kenry, Wang Y, Wu W, Zhao D, et al. Metal-organic framework as a simple and general inert nanocarrier for photosensitizers to implement activatable photodynamic therapy. Adv Funct Mater. 2018;28:1707519.

70. Zeng JY, Zou MZ, Zhang M, Wang XS, Zeng $X$, Cong $H$, et al. $\pi$-Extended benzoporphyrin-based metal-organic framework for inhibition of tumor metastasis. ACS Nano. 2018;12:4630-40.

71. Lan G, Ni K, Xu Z, Veroneau SS, Song Y, Lin W. Nanoscale metal-organic framework overcomes hypoxia for photodynamic therapy primed cancer immunotherapy. J Am Chem Soc. 2018;140:5670-3.

72. Ni K, Luo T, Lan G, Culbert A, Song Y, Wu T, et al. A nanoscale metal-organic framework to mediate photodynamic therapy and deliver CpG oligodeoxynucleotides to enhance antigen presentation and cancer immunotherapy. Angew Chem Int Ed Engl. 2019;59:1108-12.

73. Her S, Jaffray DA, Allen C. Gold nanoparticles for applications in cancer radiotherapy: Mechanisms and recent advancements. Adv Drug Deliv Rev. 2017;109:84-101.

74. Gupta A, Probst HC, Vuong V, Landshammer A, Muth S, Yagita H, et al. Radiotherapy promotes tumor-specific effector $\mathrm{CD}^{+} \mathrm{T}$ cells via dendritic cell activation. J Immunol. 2012;189:558-66.

75. Formenti SC, Demaria S. Radiation therapy to convert the tumor into an in situ vaccine. Int J Radiat Oncol Biol Phys. 2012;84:879-80.

76. Demaria S, Golden EB, Formenti SC. Role of local radiation therapy in cancer immunotherapy. JAMA Oncol. 2015;1:1325-32.

77. Schaue D, Ratikan JA, Iwamoto KS, McBride WH. Maximizing tumor immunity with fractionated radiation. Int J Radiat Oncol Biol Phys. 2012;83:1306-10.

78. Ye JC, Formenti SC. Integration of radiation and immunotherapy in breast cancer-treatment implications. Breast. 2018;38:66-74.

79. Lhuillier C, Rudqvist N-P, Elemento O, Formenti SC, Demaria S. Radiation therapy and anti-tumor immunity: exposing immunogenic mutations to the immune system. Genome Med. 2019;11:40.

80. Ni K, Lan G, Chan C, Quigley B, Lu K, Aung T, et al. Nanoscale metal-organic frameworks enhance radiotherapy to potentiate checkpoint blockade immunotherapy. Nat Commun. 2018;9:2351.
81. Munn DH, Mellor AL. Indoleamine 2,3-dioxygenase and tumor-induced tolerance. J Clin Invest. 2007;117:1147-54.

82. Lu K, He C, Guo N, Chan C, Ni K, Weichselbaum RR, et al. Chlorin-based nanoscale metal-organic framework systemically rejects colorectal cancers via synergistic photodynamic therapy and checkpoint blockade immunotherapy. J Am Chem Soc. 2016;138:12502-10.

83. Horcajada P, Gref R, Baati T, Allan PK, Maurin G, Couvreur P, et al. Metal-organic frameworks in biomedicine. Chem Rev. 2012;112:1232-68.

84. Tamames-Tabar C, Cunha D, Imbuluzqueta E, Ragon F, Serre C, Blanco-Prieto MJ, et al. Cytotoxicity of nanoscaled metal-organic frameworks. J Mater Chem B. 2014:2:262-71.

85. Oh H, Li T, An J. Drug release properties of a series of adenine-based metal-organic frameworks. Chemistry. 2015;21:17010-5.

86. Taylor-Pashow KML, Della Rocca J, Xie Z, Tran S, Lin W. Postsynthetic modifications of iron-carboxylate nanoscale metal-organic frameworks for imaging and drug delivery. J Am Chem Soc. 2009;131:14261-3.

87. Horcajada P, Chalati $T$, Serre C, Gillet B, Sebrie C, Baati $T$, et al. Porous metal-organic-framework nanoscale carriers as a potential platform for drug delivery and imaging. Nat Mater. 2010;9:172-8.

88. Bellido E, Guillevic M, Hidalgo T, Santander-Ortega MJ, Serre C, Horcajada P. Understanding the colloidal stability of the mesoporous MIL-100(Fe) nanoparticles in physiological media. Langmuir. 2014;30:5911-20.

(i) Open Access This article is licensed under a Creative Commons Attribution 4.0 International License, which permits use, sharing, adaptation, distribution and reproduction in any medium or format, as long as you give appropriate credit to the original author(s) and the source, provide a link to the Creative Commons license, and indicate if changes were made. The images or other third party material in this article are included in the article's Creative Commons license, unless indicated otherwise in a credit line to the material. If material is not included in the article's Creative Commons license and your intended use is not permitted by statutory regulation or exceeds the permitted use, you will need to obtain permission directly from the copyright holder. To view a copy of this license, visit http://creativecommons. org/licenses/by/4.0/.

(c) The Author(s) 2020 